\title{
Adaptive Prediction Model Accuracy in the Control of Residential Energy Resources
}

\author{
Rudy R. Negenborn, Michiel Houwing, Bart De Schutter, and Hans Hellendoorn
}

\begin{abstract}
With the increasing use of distributed energy resources and intelligence in the electricity infrastructure, the possibilities for minimizing costs of household energy consumption increase. Technology is moving toward a situation in which automated energy management systems could control domestic energy generation, storage, and consumption. In previous work we have proposed a controller based on model predictive control for controlling an individual household using a micro combined heat and power plant in combination with heat and electricity storages. Although the controller provides adequate performance in computer simulations, the computational time required to determine which actions to take can be significant, due to the precise predictions made over a long prediction horizon. In this paper we propose to make the computations less time consuming by coarsening the quality of the predictions made over the prediction horizon by decreasing their time resolution. In simulation studies we illustrate the performance of the proposed approach.
\end{abstract}

\section{INTRODUCTION}

\section{A. Distributed energy resources}

$\mathrm{D}$ ISTRIBUTED energy resources, comprising distributed power generation, distributed energy storage, and load management options, can play a crucial role in supporting policy objectives as electricity market liberalization, mitigating climate change, increasing the amount of electricity generated from renewable sources and enhancing energy savings $[1,2]$. A wide body of literature states that distributed generation of electricity, e.g., via photo-voltaics, wind turbines, or micro combined heat and power plants $(\mu \mathrm{CHP})$, has a good chance of pervading the electricity infrastructure in the future (see, e.g. [3, 4]). Also, several electricity storage technologies are under development (such as lithium-ion batteries, plug-in hybrid electric vehicles [5], and demand-side management options are foreseen for the future power system [6].

This work is supported by the BSIK project "Next Generation Infrastructures (NGI)", by the project "Multi-agent control of large-scale hybrid systems" (DWV.6188) of the Dutch Technology Foundation STW, the European 6th Framework Network of Excellence "HYbrid CONtrol: Taming Heterogeneity and Complexity of Networked Embedded Systems (HYCON)", contract number FP6-IST-511368, and the Delft Research Center Next Generation Infrastructures.

R.R. Negenborn, B. De Schutter, and J. Hellendoorn are with the Delft Center for Systems and Control of the Delft University of Technology, Mekelweg 2, 2628 CD Delft, The Netherlands. B. De Schutter is also with the Marine and Transport Technology department of the Delft University of Technology. M. Houwing is with the Faculty of Technology, Policy and Management of the Delft University of Technology, Jaffalaan 5, 2628 BX Delft, The Netherlands. (Corresponding author R.R. Negenborn; e-mail: r.r.negenborn@tudelft.nl; phone: +31 15 2786524; fax: +31 15 2786679).
Applying distributed generation and storage technologies at customer sites has key economic and environmental potentials. Specific potential lies in the opportunity to locally utilise the waste heat from the conversion of primary fuels into electricity by combined heat and power systems. Consequently, there has been significant progress toward developing small combined heat and power systems $(\mathrm{kW}$ scale), so-called micro-CHP or $\mu$ CHP systems, based on a Stirling engine, an internal combustion engine, a gas turbine, or fuel cell conversion technology [7]. E.g., Stirling $\mu$ CHP systems are expected to pervade the Dutch market substantially in the short- to mid-term [8]. In The Netherlands, these Stirling systems target the housing market segment of system replacements and are probably not meant for newly built houses as these have too little heat demand. The matured Dutch market is expected to comprise of around 300.000 units per year. For several other countries (e.g., UK, Germany, and Japan) $\mu \mathrm{CHP}$ technology is also expected to play a significant role [7].

The introduction of such distributed energy resources together with the introduction of more information and communication technology in the electricity system provides interesting and novel automated demand response opportunities at the domestic user level. Households thereby become more active end-users of electricity. They can devise new contractual arrangements with suppliers and/or network managers, thereby becoming more independent in terms of energy usage.

\section{B. Model predictive control}

In order to exploit the increased operational freedom of households, in [9] we proposed a controller for household energy flow optimization. The controller controls a household that has the capability of generating its own power with a $\mu \mathrm{CHP}$ unit, that can store heat and electricity, and that can trade electricity with an external energy supplier. The controller uses the control technique Model Predictive Control (MPC) [10] and has the task to automatically determine which actions should be taken in order to minimize the operational costs of fulfilling residential electricity and heat requirements subject to operational constraints. The controller uses an MPC strategy such that it can:

- take into account the decision freedom due to heat and electricity storage possibilities;

- incorporate predictions on residential electricity and heat demands; 
- incorporate models of the dynamics and constraints of installed generators and storages.

MPC is based on solving at each control step an optimization problem over a prediction horizon subject to system dynamics, an objective function, and constraints on states, actions, and outputs. At each control step the optimization yields a sequence of actions optimizing expected system behavior over the prediction horizon. Actions (control inputs) are implemented by the controller on the system until the next control step, after which the procedure is repeated with new system measurements. Due to the prediction horizon an MPC controller can take benefit of knowledge that it may have over the future, such as predicted energy demand based on historical data of energy consumption patterns.

\section{Aim and outline of this paper}

Although the MPC controller proposed in [9] chooses actions that optimize the energy costs for the household in simulation studies, the time that is required to find these actions can be considerable. The large amount of time required is due to the large amount of computations required to solve the MPC optimization problem at each control step. The aim of this paper is to reduce the computational burden of the approach presented in [9]. In this paper we propose an approximation of the original MPC optimization problem which yields a significant reduction in required computation time, while keeping the performance loss due to this reduction limited.

This paper is organized as follows. In Section II we shortly describe the main components and assumptions of the system under study. In Section III we propose the control objective and constraints of the approximation of our MPC formulation. In Section IV the performance of the proposed approach is illustrated through simulation studies.

\section{SYSTEM DESCRIPTION}

The analysis in this paper focuses on the system as shown in Fig. 1. The system consists of a household that interacts with its energy supplier. Energy trading flows are present between the household and the supplier as shown. The household has full control over its distributed energy resources and there is no interaction with other households regarding electrical energy trade.

The household fulfils its electricity and heat consumption requirements through several alternative energy supply and consumption means. The $\mu \mathrm{CHP}$ unit installed in the household is based on Stirling technology [7]. The unit consists of a Stirling engine prime mover, conversion 1, and an auxiliary burner, conversion 2, which can provide additional thermal power. The Stirling engine converts natural gas $\left(f_{l}\right)$ into electrical energy $(g)$ and heat $\left(h_{1}\right)$. The heat is supplied to a central heat storage in the form of hot water, of which the energy content is indicated by $h_{\mathrm{s}}$. The auxiliary burner also converts natural gas $\left(f_{2}\right)$ in providing

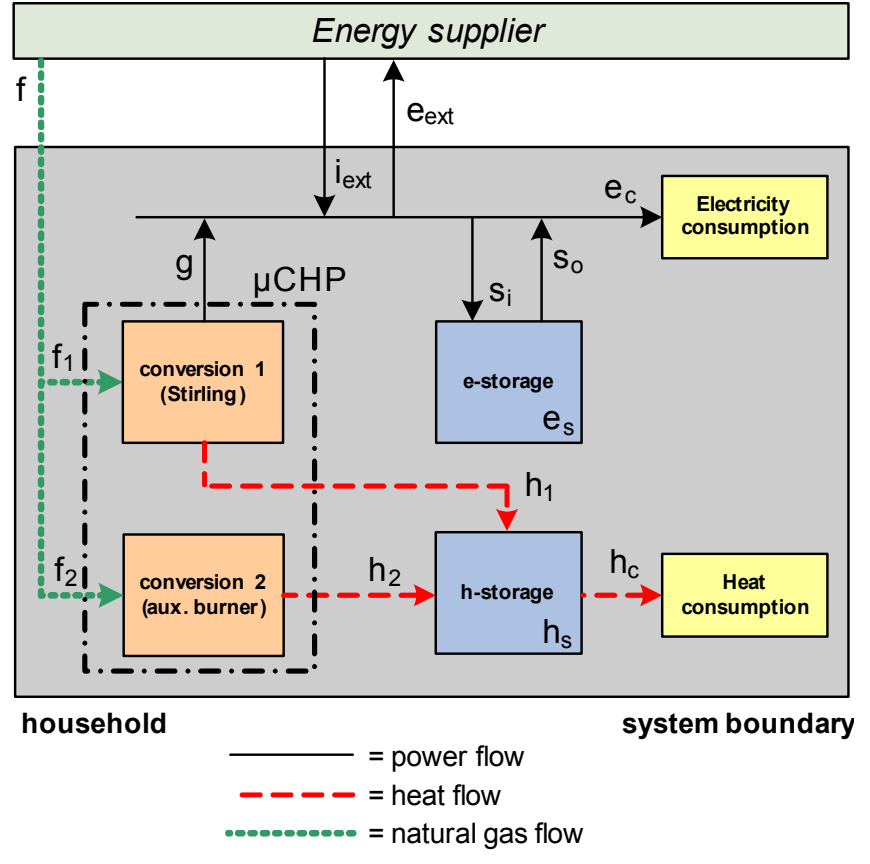

Fig. 1. Conceptual overview of the system under study.

the additional heat $\left(h_{2}\right)$. Heat consumption $\left(h_{\mathrm{c}}\right)$ is taken from the heat storage. Electrical energy can be stored in a battery $\left(e_{\mathrm{s}}\right)$ (e.g., a lithium-ion battery). In addition, electrical energy can flow to and from the battery, represented in Fig. 1 by $\left(s_{\mathrm{i}}\right)$ and $\left(s_{\mathrm{o}}\right)$ respectively. Locally generated electrical energy can be used directly by the household $\left(e_{\mathrm{c}}\right)$, it can be stored, or it can be sold to the supplier $\left(e_{\text {ext }}\right)$. Electrical energy can also be imported from the supplier $\left(i_{\mathrm{ext}}\right)$. The supplier thus sells primary fuel $\left(f=f_{1}+f_{2}\right)$ for fuelling the $\mu \mathrm{CHP}$ unit as well as additionally required import electrical energy for the household. The supplier buys any electrical energy that the household would like to feed back for a feedback tariff.

Note that we consider one large heat storage from which all heat, for domestic hot water as well as for space heating purposes, can be extracted. Such a heat storage system is currently being marketed by a manufacturer in the UK, see [11]. One storage allows us to combine space heating and domestic hot water demand, which is more convenient for the system modeling. Note also that we consider only a single heat consumption block. Buildings exhibit dynamic heat consumption behavior, depending on, among others, solar radiation, occupancy, the in-house heating system configuration and building characteristics. By making use of the heat storage from which the needed heat for the household is extracted, the exact heat demand pattern as represented by the single heat consumption block does not have to be known and it can therefore be aggregated into time blocks of relatively low resolution (15 $\mathrm{min}$ ) compared to the actual dynamic changes in heat demand.

A description of additional assumptions can be found in $[1,9]$. 


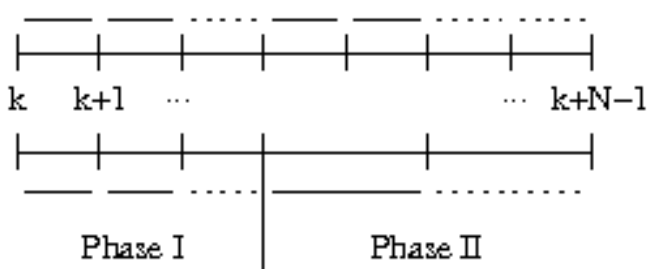

Fig. 2. Illustration of the different simulation time step resolutions considered over a prediction horizon. The top part of the figure indicates the steps used in the original approach, i.e., equidistant simulation time steps over the full prediction horizon. The bottom part indicates how in our approach for Phase I small simulation time steps are considered, while for Phase II several simulation time steps are aggregated to form larger simulation time steps.

\section{MPC FORMULATION}

In [9], a MPC controller is proposed for control of the household just described. The objective of the MPC controller is to minimize the daily operational costs of residential energy use. The MPC controller employs a control step size of 15 minutes, meaning that every 15 minutes the controller determines which action to take next. To determine which action to take, the controller makes predictions over a number of simulation steps, referred to as the prediction horizon. At each control step the controller performs the following steps:

1. Make a measurement of the current state of the household, involving measurements of the current energy levels in the heat and electricity storage.

2. Solve the MPC optimization problem, which involves finding over a prediction horizon of $N$ simulation steps of 15 minutes the actions that lead to the best predicted performance over the prediction horizon.

3. Implement the actions found until the next control step.

The MPC controller makes predictions and determines which actions to take at each control step in the prediction horizon. In a sense the controller of [9] performs long-term detailed planning of its actions at each decision step. Although this gives at each control step the best decision over the full prediction horizon, the computations involved in finding this decision can be considerable. To decrease the computational load, instead of computing an action for each time simulation time step of the prediction horizon, in this paper we propose the combination of short-term detailed planning and long-term less detailed planning. This is expected to increase computational speed, while still providing adequate control performance.

\section{A. Short-term detailed and long-term rough predictions}

The idea is to decrease the computational requirements by using a more abstract model of the system for predictions made further in the future. The abstract models are obtained by decreasing the resolution of the simulation time steps considered in the prediction horizon. Hence, whereas in the original approach equal simulation time steps of 15 minutes are considered over the complete prediction horizon, here we propose to increase the duration of simulation time steps within the horizon. Such an approach yields a reduction in the number of equations and variables involved in the optimization problem (in particular in terms of integervalued variables), and is therefore expected to yield fewer computations. Under the assumption that events appearing further away in the future have a smaller influence on control performance than events appearing soon, the performance loss should be small.

In order to obtain a combination of short-term detailed planning and long-term, less-detailed, planning, the prediction horizon is divided into two parts, and in each part of the horizon a different prediction model is used. For the first part a detailed, exact model is used. For the second part a less detailed model is used. In the following, we refer to the two parts of the prediction horizon as Phases I and Phase II. In Phase I, a simulation time step size of 15 minutes is taken, which gives an exact representation of the dynamics of the modeled system. In Phase II, a simulation time step size of 1 hour is taken. Fig. 2 illustrates the decreasing simulation time step resolution further in the prediction horizon. The system accepts new actions every 15 minutes.

Below we discuss how to formulate the prediction models and the control objectives for the different phases within the prediction horizon.

\section{B. System model formulation}

In principle, there are various ways in which the more abstract model for Phase II could be derived from the detailed model. We develop the abstract model based on expert knowledge of the system. As will be shown, the prediction model designed in this way for Phase II gives good system performance and provides a basis for future comparison with possible other models.

Our starting point is the model developed previously in $[1,9]$. In Phase I the model that exactly represents the household is used. For Phase II, as pointed out, larger simulation time steps are used. The model for Phase II is obtained by adapting some of the equations of the model for Phase I. The adaptation mainly entails the removal of binary variables to continuous variables, the rewriting of some constraints as to present system behavior when considering the larger simulation time steps and aggregating and averaging of simulation input data (described below).

We first describe the model for Phase I and then describe how the model for Phase II differs from this model.

\section{1) Model for Phase I}

The model for Phase I that we have used in this paper is similar to the model described in [9]. It is worthwhile to describe it here as well, as then the differences in modeling the system in the different phases of the prediction horizon 
can be made clear. Define the binary variables $v_{k}^{\text {CHP }}$ and $v_{k}^{\text {aux }}$, which indicate whether the installed $\mu \mathrm{CHP}$ prime mover and auxiliary burner are in operation at a specific time step $k$. In addition, the binary variables $u_{\mathrm{up}, k}^{\mathrm{CHP}}, u_{\mathrm{down}, k}^{\mathrm{CHP}}$ and $u_{\mathrm{up}, k}^{\text {aux }}, u_{\text {down }, k}^{\text {aux }}$ are start-up and shut-down indicators for the $\mu$ CHP prime mover and auxiliary burner, respectively, at time step $k$.

An electric energy balance has to be satisfied relating the power output of the Stirling engine, the input and output power flows of the electricity storage, the electricity consumption, and electricity exchanged with the energy supplier. This power balance is given by:

$$
g_{k}+i_{\mathrm{ext}, k}+s_{\mathrm{o}, k}-e_{\mathrm{ext}, k}-s_{\mathrm{i}, k}-e_{\mathrm{c}, k}=0,
$$

where $g_{k}=\eta_{\mathrm{e}} \cdot f_{1, k}$, with $\eta_{\mathrm{e}}$ the electric efficiency of the Stirling engine. The power output of the Stirling engine can be modulated between part load and full load, which is modeled by the constraints:

$$
\begin{aligned}
& f_{1, k} \leq v_{k}^{\mathrm{CHP}} \cdot f_{1, \max } \\
& f_{1, k} \geq v_{k}^{\mathrm{CHP}} \cdot f_{1, \text { part }},
\end{aligned}
$$

where $f_{1, \max }$ and $f_{1, \text { part }}$ are the fuel consumption at part and full load. For the Stirling engine there is also a minimal operation time and a minimum down time. The constraints that force the prime mover to stay in operation until this minimum has been reached are:

$$
v_{k+n}^{\mathrm{CHP}} \geq u_{\mathrm{up}, k}^{\mathrm{CHP}}, n=0, \ldots, t_{\mathrm{up}}-1,
$$

where $t_{\text {up }}$ is the minimum number of simulation time steps that the prime mover has to stay in operation. The constraints that force the prime mover to stay out of operation during down-time are:

$$
1-v_{k+r}^{\mathrm{CHP}} \geq u_{\mathrm{down}, k}^{\mathrm{CHP}}, r=0, \ldots, t_{\text {down }}-1,
$$

where $t_{\text {down }}$ is the minimum number of simulation time steps that the prime mover has to stay out of operation.

The fuel consumption of the auxiliary burner is restricted to lie within:

$$
v_{k}^{\text {aux }} \cdot f_{2, \min } \leq f_{2, k} \leq v_{k}^{\text {aux }} \cdot f_{2, \text { max }},
$$

where $f_{2, \min }$ and $f_{2 \text {,max }}$ are the minimal and maximum fuel consumption of the auxiliary burner.

The electrical energy and heat stored should be between minimum and maximum values:

$$
\begin{aligned}
& e_{\mathrm{s}, \text { min }} \leq e_{\mathrm{s}, k} \leq e_{\mathrm{s}, \max } \\
& h_{\mathrm{s}, \min } \leq h_{\mathrm{s}, k} \leq h_{\mathrm{s}, \max },
\end{aligned}
$$

where $e_{\mathrm{s}, \min }$ and $e_{\mathrm{s}, \max }$ are minimum and maximum energy levels of the battery, and $h_{\mathrm{s}, \min }$ and $h_{\mathrm{s}, \max }$ are minimum and maximum energy levels of the heat storage.

The electricity flows to and from the battery are limited by an assumed battery charge or discharge time of half an hour [5]. Hence, in 15 minutes the battery can be maximally charged or discharged with an amount equal to half the total storage capacity. Therefore, the constraint limiting the flows to and from the battery is given by:

$$
s_{\mathrm{i}, k}+s_{\mathrm{o}, k} \leq 0.5 \cdot e_{\mathrm{s}, \max },
$$

where $e_{\mathrm{s}, \max }$ is the maximum energy that can be stored in the battery. At each time step $k$ electrical energy can either only be imported from or only be exported to the external energy supplier. Constraints on the import and export power flows are therefore:

$$
\begin{aligned}
& e_{\mathrm{ext}, k} \leq \eta_{\mathrm{e}} \cdot f_{1, k}+s_{\mathrm{o}, k} \\
& e_{\mathrm{ext}, k} \leq x_{\mathrm{e}, k} \cdot P_{\max } \\
& i_{\mathrm{ext}, k} \leq e_{\mathrm{c}, k}+s_{\mathrm{i}, k} \\
& i_{\mathrm{ext}, k} \leq x_{\mathrm{i}, k} \cdot P_{\max } \\
& x_{\mathrm{i}, k}+x_{\mathrm{e}, k} \leq 1,
\end{aligned}
$$

where $P_{\max }$ is the maximum power flow allowed $(2 \mathrm{~kW})$ through the physical connection between the household and the external network, $x_{\mathrm{e}, k}$ and $x_{\mathrm{i}, k}$ are auxiliary binary variables indicating whether electrical energy is imported or exported

The heat in the heat storage changes over time depending on the heat consumption and generation. The dynamics of the heat storage are modeled by:

$$
h_{\mathrm{s}, k+1}=h_{\mathrm{s}, k}+h_{1, k}+h_{2, k}-h_{\mathrm{cp}, k},
$$

where $h_{1, k}=\left(\eta_{\text {tot }}-\eta_{\mathrm{e}}\right) \cdot f_{1, k}, h_{2, k}=\eta_{\mathrm{tot}} \cdot f_{2, k}$, and $\eta_{\text {tot }}$ is the total efficiency of the $\mu$ CHP unit. Similarly, the dynamics of the electricity storage are modeled by:

$$
e_{\mathrm{s}, k+1}=e_{\mathrm{s}, k}+s_{\mathrm{i}, k}-s_{\mathrm{o}, k} \text {. }
$$

In order to let the modeled energy conversion units function as they should, the binary variables $v_{k}^{\mathrm{CHP}}, u_{\mathrm{up}, k}^{\mathrm{CHP}}$, and $u_{\mathrm{down}, k}^{\mathrm{CHP}}$ on the one hand, and $v_{k}^{\mathrm{aux}}, u_{\mathrm{up}, k}^{\mathrm{aux}}$, and $u_{\mathrm{down}, k}^{\text {aux }}$ on the other, have to be linked. The relations between these variables are:

$$
\begin{aligned}
& v_{k}^{\mathrm{CHP}}-v_{k-1}^{\mathrm{CHP}}=u_{\mathrm{up}, k}^{\mathrm{CHP}}-u_{\mathrm{down}, k}^{\mathrm{CHP}} \\
& v_{k}^{\mathrm{aux}}-v_{k-1}^{\mathrm{aux}}=u_{\mathrm{up}, k}^{\mathrm{aux}}-u_{\mathrm{down}, k}^{\mathrm{aux}} \\
& u_{\mathrm{up}, k}^{\mathrm{CHP}}+u_{\mathrm{down}, k}^{\mathrm{CHP}} \leq 1 \\
& u_{\mathrm{up}, k}^{\mathrm{aux}}+u_{\mathrm{down}, k}^{\mathrm{aux}} \leq 1 .
\end{aligned}
$$

\section{2) Model for Phase II}

The prediction model used for Phase II is derived from the detailed model for Phase I. The main differences between the original prediction model and the model for Phase II are the following:

- The start-up and shut-down behavior of the Stirling engine and the auxiliary burner is modeled differently and therefore constraints (4) and (5) are not present.

- We treat the auxiliary burner power output as a continuous variable. The binary variable $v_{\mathrm{aux}, k}$ therefore becomes unnecessary.

- Because the simulation time steps span longer time intervals, there can now be import and export of power in the same simulation time step. Hence, constraint (11), (13), and (14) are absent.

- In the energy balance equations, aggregated electricity and heat demand values are used, obtained by aggregating the 15-minute demand data to obtain data per hour for Phase II. 
Due to the above abstractions the integer variables $u_{\mathrm{up}, k}^{\mathrm{CHP}}, u_{\mathrm{down}, k}^{\mathrm{CHP}}, u_{\mathrm{up}, k}^{\mathrm{aux}}, u_{\mathrm{down}, k}^{\mathrm{aux}}, v_{k}^{\mathrm{aux}}, x_{\mathrm{i}, k}, x_{\mathrm{e}, k}$ become obsolete and are removed from the model. The equations of the system model that change substantially for Phase II are described below.

Since if the Stirling engine runs, its energy output should be between the output value corresponding with the minimal up-time and the maximum value, instead of equations (2) and (3) for the Stirling engine operation, the following constraints are used:

$$
\begin{aligned}
& f_{1, k} \geq t_{\text {up }} \cdot v_{k}^{\mathrm{CHP}} \cdot f_{1, \text { part }} \\
& f_{1, k} \leq r_{\text {II }} \cdot v_{k}^{\mathrm{CHP}} \cdot f_{1, \max },
\end{aligned}
$$

where $r_{I I}$ is the number of time steps that are considered aggregated in the model of Phase II. The auxiliary burner constraint (6) is changed to:

$$
r_{\text {II }} \cdot f_{2, \text { min }} \leq f_{2, k} \leq r_{\text {II }} \cdot f_{2, \text { max }} \text {. }
$$

The constraint on the power flows to and from the battery change from (9) to:

$$
s_{\mathrm{i}, k}+s_{\mathrm{o}, k} \leq r_{\mathrm{II}} \cdot 0.5 \cdot e_{\mathrm{s}, \max } \cdot
$$

Finally, the constraint on the power import and export change from (11), (13) and (14) to:

$$
e_{\mathrm{ext}, k}+i_{\mathrm{ext}, k} \leq r_{\mathrm{II}} \cdot P_{\max } \text {. }
$$

The prediction models for Phases I and II are coupled to each other. The model for Phase I requires the measurement of the current state. Using the model for Phase I, predictions are made for $N_{\text {I }}$ prediction steps of 15 minutes. From that moment, the model of Phase II is used to make $N_{\text {II }}$ prediction steps of 60 minutes.

\section{Control objectives}

1) Objectives for Phase I

For Phase I, costs are computed for each simulation time step of 15 minutes. The operational costs depend on the price $p_{\mathrm{f}}$ for gas consumption, the price $p_{\mathrm{i} \text {,ext }}$ for importing electricity and the price $p_{\mathrm{e}, \mathrm{ext}}$ at which electricity can be sold. The cost function for control step $k$ with a prediction horizon of $N_{\mathrm{I}}$ is therefore defined as:

$$
\begin{aligned}
& J(\cdot)= \\
& \sum_{m=0}^{N_{\mathrm{l}}-1}\left(\left(f_{1, k+m}+f_{2, k+m}\right) \cdot p_{\mathrm{f}}+i_{\mathrm{ext}, k+m} \cdot p_{\mathrm{i}, \mathrm{ext}, k+m}-e_{\mathrm{ext}, k+m} \cdot p_{\mathrm{e}, \mathrm{ext}}\right) .
\end{aligned}
$$

\section{2) Objectives for Phase II}

For Phase II the control objective function is similar to (26). The electricity prices differ, however, as in Phase II, average prices for electricity import and export are taken over the aggregated simulation time steps.

\section{Simulations}

In this section we illustrate the performance of the proposed approach with simulation experiments. We focus on the relation between the reduction in the computational load for the MPC controller and the accompanying change

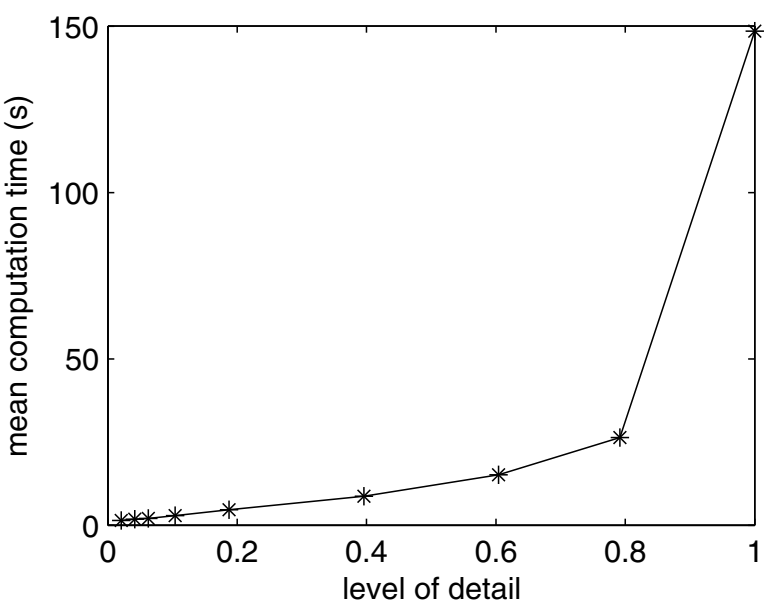

Fig. 3. Mean computation time required (s) over all control steps for varying levels of detail considered in the prediction horizon.

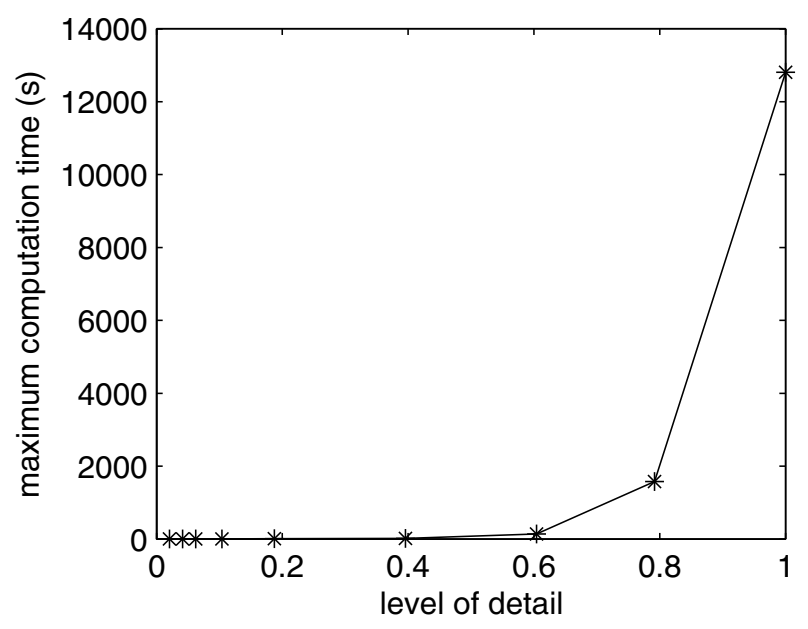

Fig. 4. Maximum computation time required (s) over all control steps for varying levels of detail considered in the prediction horizon.

in system performance.

\section{A. Implementation}

We have implemented the described system and MPC controller in Matlab 7.4. The optimization problem that has to be solved at each decision step is a mixed-integer linear programming problem, due to the presence of continuousvalued and binary-valued variables in combination with the lienar objective function and constraints. We use the stateof-the-art mixed-integer solver CPLEX v10 through the Tomlab interface to Matlab to solve the problem.

\section{B. Simulation setup}

We consider a simulation period of one week in a winter season. Heat demand and electricity demand patterns, as well as prices for gas and electricity are assumed given on a per quarter basis, similarly as in [9]. The import and export prices are taken equal to each other and vary per hour. The starting values for the simulation of the system are for $k=1$ taken as:

$$
\begin{aligned}
& v_{1}^{\mathrm{CHP}}=v_{1}^{\mathrm{aux}}=u_{\mathrm{up}, 1}^{\mathrm{CHP}}=u_{\mathrm{down}, 1}^{\mathrm{CHP}}=u_{\mathrm{up}, 1}^{\mathrm{aux}}=u_{\mathrm{down}, 1}^{\mathrm{aux}}=0, \\
& e_{\mathrm{s}, 1}=e_{\mathrm{s}, \min }, h_{\mathrm{s}, 1}=h_{\mathrm{s}, \min } .
\end{aligned}
$$




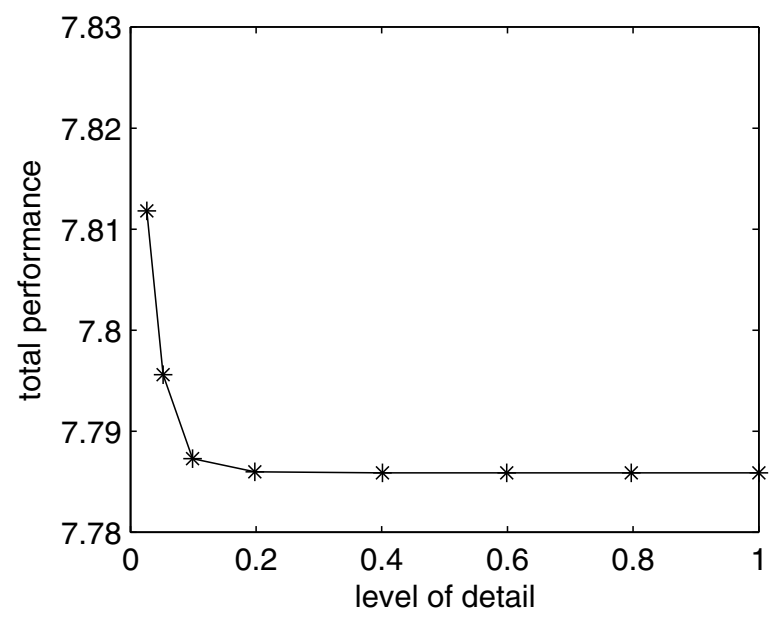

Fig. 5. Total cost over full simulation of one week for varying levels of detail consi dered in the prediction horizon.

We are interested in observing the reduction in computational load as the predictions made over the prediction horizon are made less precisely. We do this by varying for a specific prediction horizon length the number of simulation steps that is considered in detail (modeled with the Phase I model) as well as the number of simulation steps that is considered in less detail (modeled with the Phase II model). As a prediction horizon we take two days, i.e., 192 simulation steps of 15 minutes. We start with computing the time required and performance obtained when making 192 detailed simulation steps and thus do not employ aggregated simulation steps in the prediction horizon. We then gradually decrease the detailed part of the prediction horizon by replacing the detailed simulation steps with aggregated simulation steps.

\section{Results}

Fig. 3 illustrates the mean computational time required at each control step, when simulating for one full week, and while varying the degree of detail considered by the controller over the prediction horizon. We define this degree of detail as the number of prediction steps considered in detail with the Phase I model devided by the total number of prediction steps in the prediction horizon, i.e., 192. We clearly observe that as the degree of detail is decreased, the mean computational time required decreases significantly. Fig. 4 shows the maximum computational time required over all control steps for varying degrees of detail. For larger degrees of detail, the computational time required is significantly larger than the control step length.

Fig. 5 illustrates the change in the total performance, i.e., the performance over the full simulation of one week in terms of operational costs of energy use, as we vary the degree of detail considered over the prediction horizon. We observe that the total performance decreases only slightly with the decrease in detail. Hence, these simulations clearly illustrate that the approach proposed in this paper has the potential to yield a significant reduction in computational time required, while only giving a slight reduction in performance.

\section{CONCLUSIONS AND FUTURE RESEARCH}

In this paper we have discussed reduction of computational requirements of a model predictive control (MPC) controller for household optimization. We have proposed to reduce the computational requirements of the controller by coarsening the predictions made over the prediction horizon. Our approach relies on using a prediction model that makes predictions at more coarse time intervals as prediction steps further in the prediction horizon are considered. In experiments using a simulation study we have illustrated the reduction in computational requirements and the relatively low reduction in performance.

Future research should focus on more structured approaches of setting up the approximations of the detailed model, determining if it is beneficial to include a further coarsening of the prediction horizon, and analyzing the effect of the horizon length on the performance. In addition, the robustness of the MPC controller against uncertainty in measurements and predictions of energy consumption patters should be investigated. Furthermore, the implementability of the proposed approach using embedded hardware should be addressed to make the step to a practical implementation.

\section{REFERENCES}

[1] M. Houwing, A. Ajah, P. W. Heijnen, I. Bouwmans, and P. M. Herder, "Uncertainties in the Design and Operation of Distributed Energy Resources: The Case of Micro-CHP Systems". To appear in Energy The International Journal, 2008.

[2] M. Houwing, P. W. Heijnen, and I. Bouwmans, "Socio-Technical Complexity in Energy Infrastructures - Conceptual Framework to Study the Impact of Domestic Level Energy Generation, Storage and Exchange". In Proc. of the IEEE International Conference on Systems, Man and Cybernetics, Taipei, Taiwan, October 2006.

[3] A. Chambers, S. Hamilton, and B. Schnoor, Distributed Generation: A Nontechnical Guide. Tulsa, Oklahoma, US: Penn Well Corporation, 2001.

[4] N. Jenkins, R. Allan, P. Crossley, D. Kirschen, and G. Strbac, Embedded Generation. London, UK: The Institution of Electrical Engineers, 2000.

[5] E. Lysen, S. Van Egmond, and S. Hagedoorn, "Opslag van elektriciteit: Status en toekomstperspectief voor Nederland" (in Dutch). Utrecht Centrum voor Energieonderzoek - SenterNovem NEO 0268-05-05-01-002, September 2006.

[6] S. D. Braithwait, "Real-Time Pricing and Demand Response Can Work within Limits". In Natural Gas \& Electricity, vol. 21, 2005.

[7] M. Pehnt, M. Cames, C. Fischer, B. Praetorius, L. Schneider, K. Schumacher, and J. Vob, Micro Cogeneration: Towards Decentralized Energy Systems. Berlin: Springer, 2006.

[8] A. De Jong, E. J. Bakker, J. Dam, and H. Van Wolferen, "Technisch energie- en CO2-besparingspotentieel van micro-wkk in Nederland (2010-2030)" (in Dutch). Werkgroep Decentraal, onderdeel van Platform Nieuw, July 2006.

[9] M. Houwing, R. R. Negenborn, P. W. Heijnen, B. De Schutter, and J. Hellendoorn, "Model predictive, least cost control of residential energy resources when applying $\mu \mathrm{CHP} "$. In Proc. of IEEE PowerTech 2007, Lausanne, Switzerland, July 2007.

[10] J. M. Maciejowski, Predictive Control with Constraints. Harlow, England: Prentice Hall, 2002.

[11] http://www.gledhill.net, "Gledhill water storage - UK", 2007. 